\title{
Long-Term Multi-criteria Improvement Planning
}

\author{
J.P. Hubinont, Y. De Smet
}

June 3, 2021

\begin{abstract}
This paper proposes a novel framework to support the generation of strategies for multi-criteria longterm improvement. It can be applied to general preference models but it is illustrated in this article on a Multi-Attribute Value Theory model. The novel contributions to the literature are twofold. Firstly, the framework addresses the issue of resistance to change that may arise during the implementation of a strategy. It constrains a step of improvement to be focused on a single criterion, and minimizes the intensity of operational changes. Secondly, it addresses the realism of the improvement scenarios by treating three types of structural dependencies differently: the positive synergies, the negative synergies and the bottlenecks. The scenarios are generated by finding a set of efficient solutions to a shortest path problem in a graph whose edges represent possible steps of improvement. Each edge is characterized by an increase of rank or level and two penalty functions relating to the difficulty of its execution, one representing a risk associated to bottleneck mechanisms and the other to operational change relative to a previous edge. A case-study using the Shanghai Academic Ranking of World Universities is presented in order to illustrate how this framework could be useful to generate a sequence of strategic actions for the Université libre de Bruxelles.
\end{abstract}

\section{Introduction}

Multi-criteria improvement planning is a complex problem that involves a group of stakeholders who are concerned with the performance evolution of a given entity (such as a company, a country, an administration, etc.).

The stakeholders can be divided into three categories. Firstly, the decision maker(s), who plan and study how to modify the performances of the considered entity. Secondly, the worker(s), who are involved in the 
operational implementation of the planning. Thirdly, the receiver(s), on whom the decision has an influence and who possibly have, in turn, a role to play in the decision acceptation and implementation. Due to the resistance to change that can arise from the workers and the receivers (Dent \& Goldberg, 1999), (Bovey \& Hede, 2001 ), improvement planning should be studied with the will to limit systemic changes (short-term operational changes) for a given strategic change (long-term vision that acts as a constraint for the improvement) (McNair \& Leibfried, 1993). As a corollary, McNair \& Leibfried (1993) advocate that "systemic change is possible but it cannot be done in every part of the organization at once" and that "changes have to be focused on achieving a new vision of the organization that will replace existing practices and traditions". This observation constitutes one dimension of the complexity of improvement planning faced by the decision makers.

In addition to this first difficulty, the decision makers must be able to model the problem in an appropriate way. This involves the definition of indicators which will be used to evaluate the entity and its possible evolution based on available data or on the judgment of experts. As explained by Roy (1985), a crisp representation of the nature by a limited amount of indicators is often a source of errors. This adds another layer of complexity for improvement planning. Moreover, if the present and the observed are already hard to model, the future is even more so. Besides, most of the time, these indicators are in conflict, i.e. it is usually impossible for a given entity to perform well on all indicators simultaneously. As a consequence, the decision makers need a methodology to be able to compare different entities with their own advantages and drawbacks in relation to each other. On top of that, they will have to decide on which indicators and to which degree they could improve the performance of the studied entity realistically and desirably.

Finally, the decision can be planned once, typically for short-term improvement planning, or planned repeatedly for a long-term evolution. This further enhances the complexity of the task.

The comparison of entities evaluated on different indicators is at the core of Multi-Criteria Decision Analysis (MCDA). In this context, tools have been developed to help reveal the preferences of the decision makers in order to properly model the problem. Different families of MCDA methods have been proposed in the literature and vary depending on the underlying assumptions related to the preference structure of the decision makers. These have been applied in different fields (Behzadian et al. 2010), including budgeting and resource allocation (Lootsma et al. 1990), geographical information systems (Ligmann-Zielinska \& Jankowski, 2012), 
health care (Georges et al. 2015), etc.

Within operational research, another field called Data Envelopment Analysis (DEA) also focuses on the comparison of entities based on multidimensional evaluations (Banker et al., 1984, Charnes et al. 1978). In this context, indicators are considered to be either inputs (that the entity consumes) or outputs (that the entity produces). Here the focus is to assess the efficiency of entities (that are called Decision Making Units) in relation to each other. Typically, the aim is to give a recommendation for an inefficient entity to become efficient (in one or several steps). DEA is strongly related to benchmarking which is a systematic search for the best practices that will help the compared entities improve and reach the best performances (Camp, 1989). If several consecutive performance modifications are suggested, it is called stepwise benchmarking. This has been introduced in order to ease the process of improvement, or to make it more realistic, with regard to the similarity of each intermediate step (see Park (2012)), to the context-dependency (see Seiford \& Zhu (2003)), or to the risk of failure (see Ghahraman \& Prior (2016)), among others (Alirezaee \& Afsharian, 2007, Estrada et al., 2009, Lim et al., 2011). Let us note that all these contributions propose that each intermediary step match exactly an observed entity's performance. Alternatively, other works allow fictitious (not observed) steps (Lozano \& Villa, 2005), (Lozano \& Calzada-Infante, 2017).

Stepwise benchmarking has mainly been studied for problems that fit the DEA assumptions (i.e. the existence of indicators that can be explicitly viewed as inputs and outputs). Less attention has been paid to problems that do not fulfill these hypotheses and that typically belong to the MCDA field. A first family of contributions to generate and plan scenarios of improvement can be found in Petrović et al. 2012, 2014. 2018b a). These approaches explicitly take into account the decision makers' preference structure. Petrović et al. (2014) introduced stepwise benchmarking to the MCDA domain, using a modified version of the ELECTRE II method (Roy, 1990) developed in Bojković et al. (2010). They propose a discrete approach to model the performance evolution of each entity into a simple measurement system. An ordered partition of the entities is built allowing to represent groups of less preferred entities (which correspond to lower layers) to preferred entities (which correspond to higher layers). They have developed an optimal algorithm to find the best path of improvement, among these layers, by minimizing the difference of the Euclidean distance between each step. In addition, they propose a sensitivity analysis to their model (Petrović et al., 2018a). 
Another recent contribution that involves multi-criteria improvement planning is Post Factum Analysis (PFA) proposed by Kadziński et al. (2016) and later applied and developed by Ciomek et al. (2018), as well as Dutta et al. (2019). It is strongly related to the robustness concern associated with a given recommendation. Among other results, the authors provide an interactive optimization method to evaluate the most realistic and attractive option (based on an individual's judgment represented by additive value functions) to improve the rank of a given entity by modifying a predefined subset of criteria. Their approach is very interesting in the context of a single step improvement. However, in the more general frame of sequential improvements, it becomes harder to manage, as the realism and feasibility of each step should be estimated by the decision maker.

The different approaches presented are tools that allow to benchmark several observed entities and to generate scenarios of improvement in the formal representation, i.e. in terms of evaluated performances, not real life actions. Regarding the practical interest in benchmarking, it has been used as a monitoring tool for cities, countries, companies, etc. Since the early 2000's, the European Union has been promoting benchmarking as a monitoring tool to help each country learn the best practices from others (Borrás \& Jacobsson, 2004), (Arrowsmith et al. 2004). Stepwise benchmarking has been applied to banks (Alirezaee \& Afsharian, 2007), printer retailers (Seiford \& Zhu, 2003), European telecommunication (Petrović et al. 2018b), and will be used in the future as the European Commission and the European Securities and Markets Authority (ESMA) strive for the regulation and stability of the financial benchmarks in the banking sector, in order to assess financial risks regarding derivatives, loans etc. ((Steven, 2018), (IOSC, 2013)). In addition, let us note that other benchmarks exist and could be used as tools for stepwise improvement: University rankings (Academic Ranking of World Universities (Billaut et al. 2010), Times Higher Education; country or city rankings (the UN's development goals for sustainability, the Environmental Performance index, the IMD smart city index), etc. In this context, the DEA assumptions (i.e. the explicit presence of inputs and outputs) are not naturally satisfied.

The tools offered to plan a sequence of improvement are mostly focused on finding similar observed entities to learn from. However, as the generated scenarios constitute a basis to build real strategic options, more attention should be paid to easing the implementation of improvement planning with regard to the stakeholders (which involves focused and limited systemic changes) as well as to easing the investigation on how 
to match improvement in the formal representation to real life actions. Moreover, for the sake of realism, the vast majority of the methods limit the possible future performances to those of other observed entities which is not always necessary and which reduces the chance of possible innovation in the generated scenarios.

We propose a theoretical framework to generate long-term multi-criteria improvement scenarios (with a planned sequence of improvement), such that each step focuses on improving one criterion at a time. Our approach intervenes at the same level as PFA, proposed by Kadziński et al. (2016) (it must be applied to a predefined preference model). However, our framework proposes guidelines to build stepwise benchmarking and provides assumptions to define a realistic evolution of performances which are not especially observed (contrary to Petrović et al. (2018b)). In this respect, this framework is illustrated with an additive Multi-Attribute Value Theory (MAVT) model. It is important to note that the guidelines could be defined differently for other families of preference methods such as ELECTRE (Figueira et al., 2005), for example. It will, indeed, be the subject of future research, which goes beyond the scope of this article.

In addition to the guidelines, a graph is created (starting from the performances of the entity to improve), the edges of which are weighted with three important characteristics to describe a long-term multi-criteria improvement scenario. The first indicator is the effort of execution if a given step risks facing a bottleneck mechanism (defined in Section 3), and should be minimized. The second is the effort of execution if a given step represents an operational change in relation to the previous step, and should also be minimized. The third is the rank or level increase and should be maximized. Finally, a representative set of efficient solutions is proposed and studied with the decision maker. Different analyses should be conducted together with the decision maker in order to discriminate the final convincing scenario(s): a scenario should be robust, realistic and desirable. We propose a very simple sensitivity analysis in the case-study presented in Section 6. However, a rigorous robustness analysis will be the subject of future research.

It is important to note that our work aims at generating and selecting several scenarios of improvement in the formal representation leading to create real life strategic action plans that would not have necessarily been conceived by a decision maker. When convincing action plans have been selected, a more precise analysis of the costs and work-load required in order to implement the strategy can be dealt with by building a strategy table as proposed by Howard (1988), for instance. Indeed, there are benchmarks which use non-measurable 
indicators (such as surveys for example in the Times Higher Education ranking, assessing the quality of each university's teaching or research). It would be difficult for a university willing to improve through this ranking to put a cost on getting better at these surveys. However, one university could be inspired by those with better survey results to match real life actions. Therefore, this framework should be used as a generator of scenarios of improvement in a formal representation of the problem with observed entities as reference for each step, in order to dig into the real life actions of these entities, and ultimately create real strategic options and apply any existing valuation models that can be found in the vast literature of portfolio selection (Salo et al., 2011) or strategic decision analysis (Howard, 1988, Montibeller \& Franco, 2011).

Finally, a software integrating this framework has been made publicly available and as an open source at http://dx.doi.org/10.17632/7cbsmm5sx9.1 in the format of a python file (MCDMBM.py) and of an executable file (MCDMBM.exe) to allow users willing to apply our framework without coding knowledge and let others modify it if needed. A configuration file (Article_cgf.txt) containing the parameter values used in Section 6, is also provided in order to be able to easily reproduce the results presented in this article (and modify it).

This article is organized as follows: Section 2 provides the notation used to describe the framework. Section 3 introduces the different guidelines and assumptions that are the basis of this work. Section 4 proposes a methodology to build a graph and find efficient solutions. Section 5 applies our framework to an MAVT preference model. Section 6 illustrates how the framework and MAVT model can be applied to generate scenarios of improvement and strategic action plans for a university attempting to improve its rank in the Academic Ranking of World Universities (ARWU).

\section{Notation}

We will use the following notation:

- $A=\left\{a_{1}, \cdots, a_{i}, \cdots, a_{m}\right\}$ - a set of observed entities called alternatives (and not actions, in order to distinguish them from real life actions);

- $a_{D M} \in A$, an alternative whose performances should be improved by a Decision Maker (DM). It is assumed that we only deal with one decision maker. Moreover, $a_{D M}$ should be considered all through 
the article as having an evolving performance;

- $G=\left\{g_{1}, \cdots, g_{j}, \cdots, g_{n}\right\}$ - a set of criteria (to be maximized without loss of generality);

- $E_{j}$, the performance scale of criterion $g_{j}$;

- $E=E_{1} \times \cdots \times E_{j} \times \cdots \times E_{n}$ - the performance space;

- $g_{j}\left(a_{i}\right)$ - the performance of an alternative $a_{i}$ on criterion $g_{j}$;

- $g\left(a_{i}\right)=\left(g_{1}\left(a_{i}\right), \cdots, g_{j}\left(a_{i}\right), \cdots, g_{n}\left(a_{i}\right)\right)$ - the performance of an alternative $a_{i}$ such that $g\left(a_{i}\right) \in E$;

- $b_{j}^{*}$ - the best reachable performance on criterion $g_{j}$ for $a_{D M}$;

- $b_{j *}$ - the worst reachable performance on criterion $g_{j}$ for $a_{D M}$;

- $p=\left(p^{1}, \cdots, p^{q}, \cdots, p^{Q}\right)$ - a path of improvement of size $Q$ where $p^{1}=g\left(a_{D M}\right)$ and $p^{q} \in E, q=2, \ldots, Q$ where $g\left(a_{D M}\right)$ is understood here as the initial performance of $a_{D M}$;

- Let $x, y \in E$. The notation $x_{-k} y$ denotes the performance of $x$ where the $k^{t h}$ component has been replaced by the $k^{\text {th }}$ component of $y$.

\section{General framework}

As stated in the introduction, we newly propose to generate paths of improvement composed of steps involving a performance improvement on only one criterion at a time. For the sake of simplicity, we will call such steps single performance improvements in the rest of the article.

As already stressed, building a realistic path of improvement is a complex task. In this context, at least four main aspects must be considered:

1. Degradation of performance: Should a degradation of performance appear in the path, it would obviously be perceived as negative by the decision maker. In the multi-criteria literature for instance, Petrović et al. (2014) forbid any degradation of performance score in their recommendation. However, it is often necessary and realistic in practice;

2. Efficiency of improvement: when a degradation of performance is allowed in an improvement path, it should be limited; 
3. Realism: An improvement should be realistic in terms of structural dependencies between criteria and in terms of resources for the decision maker;

4. Implementation: As explained, a path of improvement should support a focused vision and should be constituted by a limited amount of operational changes.

This article positions itself in line with the literature regarding the degradation of performance. As will be explained, a possible degradation of performance will be studied, but not taken into account while building the paths of improvement. Regarding realism, structural dependencies between criteria often exist under the form of positive or negative interactions but also under the form of constraints. As explained in Roy (1985) a structural dependence between criteria is the classical notion of dependence that exists in the presence of factors possibly jointly influencing several criteria. They are intrinsic to the nature of the criteria and are entirely different from the preferential dependencies also defined in Roy (1985). They are generally neither linear nor invariant. For this reason, we believe that modelling these dependencies is a task far too complex in practice for a decision maker (especially when the number of criteria increases). This is one of the arguments to learn from the similar used in benchmarking problems. It is often stated that what is observed is realistic and therefore implicitly takes structural dependencies into account (Lim et al., 2011). We suggest differentiating two types of negative structural dependencies: bottlenecks on the hand and negative synergies on the other.

A bottleneck is a blocking mechanism caused by a wrongly planned order of improvement criteria that could result in the use of resources for a process without improving (and possibly without degrading) the performance on any criterion (McNair \& Leibfried, 1993). For instance, a retail company evaluated on its market segment as well as on its average phone prices could be willing to improve on both criteria. Depending on the financial situation of the company it could reduce its prices before trying to reach more customers (with advertising for instance) if it already has enough liquidity. The risk of not doing so could result in a waste of resources in advertising, yielding a low expansion of its market segment. On the contrary, if the company is newer, it could lack liquidity and public reputation, therefore it might be a better option to firstly enlarge the market segment and later reduce the average price. The risk in this case is that if the price is reduced before enlarging the market segment, the company might lack liquidity and thus money for an efficient advertising campaign. These blocking mechanisms that require the improvement of the criteria in the right order to avoid a loss of resources are known as bottlenecks. 
A negative synergy implies that increasing the performance of one or several criteria degrades the performance of one or several other criteria. Contrary to bottlenecks, these losses of performance are sometimes necessary in order to improve globally.

These two effects should ideally be detected and treated differently. While bottlenecks should be avoided, negative synergies (which can lead to a degradation of performance) should be limited. These two types of effects will be referred to as barriers to improvement from this point on in the article. A given improvement path should be such that it faces as few unforeseen barriers to improvement as possible.

Regarding positive synergies, they will be studied in a post-analysis step with the decision maker. Therefore, we will first and foremost explain how to generate a path of improvement, then we will explain how positive synergies are studied in Section 4 .

Now that the main features of our framework have been stated, let us analyze an assumption that is commonly made in the literature: learn from the similar (Seiford \& Zhu, 2003), (Lim et al., 2011), (Park, 2012), (Ghahraman \& Prior, 2016), (Petrović et al., 2018b), etc. It could be summarized as follows:

Assumption 1 (learning from the similar) Let us consider $a_{i}, a_{j}$ in A. If $a_{i}$ is proved (by any MCDA preference model or DEA model) to perform better than $a_{j}$ and if they are similar, it is realistic to think that $a_{j}$ can improve to match the performance of $a_{i}$, without significant degradation of performance.

The notion of similarity can be defined in different ways: by the Euclidean distance as in (Park, 2012 Petrović et al. 2014), or through a clustering procedure as in (Hong et al., 1999, Ghahraman \& Prior, 2016), among others. However, this assumption has limitations. Regarding the implementation of each step, Assumption 1 generates a certain amount of indeterminacy regarding the execution of the improvement. Without further knowledge, all the modifications of performance that lead to improving $a_{j}$ in order to match the performance of $a_{i}$ are supposed to be equally realistic and are left to the judgment of the decision maker. This brings flexibility (which can be appropriate) but also indeterminacy. Moreover, focused improvement cannot be guaranteed. Indeed, the possible scenarios of improvement are limited by the hierarchy built between the observed alternatives (for more information see Alirezaee \& Afsharian (2007) for the DEA methods and Petrović et al. (2014) for the multi-criteria method). Therefore, it could be suggested 
that nearly all criteria are improved at the same time. Finally, the assumption can easily be accepted theoretically, but in practice, there will probably be some degradation of performance through negative synergies.

Let us now describe, in detail, the theoretical framework that we propose as a support to generate focused and realistic improvement paths. This framework will be defined using notation in Greek letters in order to distinguish it from the illustration of the particular MAVT preference model presented in Section 5. The general framework is based on four different steps.

Step 1 - In collaboration with the decision maker a preference model $M$ is built and defines at least a partial pre-order on $A$. We consider the following binary relations for all $a_{i}, a_{j} \in A$ :

- Outranking relation $\left(a_{i} S a_{j}\right): a_{i}$ is at least as good as $a_{j}$;

- Strict preference $\left(a_{i} P a_{j}\right): a_{i}$ is preferred to $a_{j}\left(a_{i} P a_{j} \Longleftrightarrow a_{i} S a_{j}\right.$ and $\left.a_{j} \neg S a_{i}\right)$;

- Indifference $\left(a_{i} I a_{j}\right): a_{i}$ is similar to $a_{j}\left(a_{i} I a_{j} \Longleftrightarrow a_{i} S a_{j}\right.$ and $\left.a_{j} S a_{i}\right)$;

- Incomparability $\left(a_{i} D a_{j}\right): a_{i} D a_{j} \Longleftrightarrow a_{i} \neg S a_{j}$ and $a_{j} \neg S a_{i}$.

Moreover, the model $M$ should allow to identify one of the two following pieces of information related to the set of alternatives $A^{*}=A \backslash\left\{a_{D M}\right\}$ and any fictitious or observed alternative $x$ :

1. $R\left(x, A^{*}\right)$ : The rank of $x$ in the partial pre-order on $\{x\} \cup A^{*}$, such that $R\left(x, A^{*}\right)=1$ means that $x P a_{i}^{*}$ for all $a_{i}^{*} \in A^{*}$;

2. $C\left(x, A^{*}\right)$ : The level or class in $A^{*}$ to which $x$ belongs when all the levels/classes are ordered from the best to the worst.

Obviously, when using an additive model (as any MAVT model), one can use the total order on $\{x\} \cup A^{*}$ and thus $R\left(x, A^{*}\right)$. When using outranking methods such as ELECTRE (Figueira et al. 2005), classes of alternatives can be built.

Let us note that using $R\left(x, A^{*}\right)$ or $C\left(x, A^{*}\right)$ is based on the assumption that while $g\left(a_{D M}\right)$ can evolve towards a performance $x \in E$, the other observed alternatives $a_{i} \in A^{*}$ are assumed to remain in an idling mode. Their performance remains constant. This is an unrealistic assumption used for the sake of simplicity and 
could be relaxed in future works.

Step 2 - If one considers a single performance improvement step, one should be able to find a subset $\Lambda_{k} \subseteq A$ which contains observed alternatives that can inspire a performance improvement for any $g_{k}$. These alternatives should allow $a_{D M}$ to improve globally even if it induces negative synergies while replicating their performance on $g_{k}$. We propose to adopt a pessimistic approach and consider the worst scenario. This consists in $a_{D M}$ degrading its performance on all criteria $g_{j}$ for which $a_{i}$ performs less well than $a_{D M}$ (not worse than $b_{j *}$, the worst possible performance for $a_{D M}$ ) and in improving its performance solely on $g_{k}$ (not more than $b_{k}^{*}$, the best possible performance of $a_{D M}$ on $g_{k}$ ). This worst-case scenario will be denoted $w c\left(a_{D M}, a_{i}\right)$. In order to evaluate if such a scenario would still allow $a_{D M}$ to improve globally, we suggest that $a_{i}$ belong to $\Lambda_{k}$ if the two following conditions are met:

1. $w c\left(a_{D M}, a_{i}\right) \succ_{k} a_{D M}$ (where $\succeq_{k}$ means "is at least as good as" on criterion $\left.g_{k}\right)$. Therefore this condition means that $a_{i}$ must be preferred to $a_{D M}$ considering only criterion $g_{k}$ even if its performance is limited to $\left.b_{k}^{*}\right)$;

2. $\Delta_{k}\left(a_{D M}, a_{i}\right) \geq 0$ where $\Delta_{k}: E^{2} \rightarrow \mathcal{R}$ quantifies how much the worst scenario, $w c\left(a_{D M}, a_{i}\right)$, would still be preferred to the current performance of $a_{D M}$. If $\Delta_{k}\left(a_{D M}, a_{i}\right) \geq 0$, this means that the worst scenario is at least as good as the current performance of $a_{D M}$.

These are the main constraints for the definition of $\Delta_{k}$. However, other constraints can be added depending on the preference model used and the decision maker's judgment. For instance, it could be decided that an improvement step should at least entail an increase of a given number of ranks.

Step 3 - Suppose that $\Lambda_{k}$ is built for each criterion $g_{k}$. One must select which observed alternative from $\Lambda_{k}\left(a_{D M}\right)$ could be chosen as an intermediate benchmark. By the same token as Assumption 1 . we propose to find the most similar alternative to $a_{D M}$ on criterion $g_{k}$, denoted $\beta_{k}\left(a_{D M}\right)$. It is defined as follows:

$$
\beta_{k}(x)=\underset{\forall a_{i} \in \Lambda_{k}(x)}{\arg \min } g_{k}\left(a_{i}\right)
$$

If there are several possible candidates, one must select appropriate discrimination rules. We suggest a lexicographical rule in Appendix $\mathrm{A}$ if such a case happens. However, this rule is flexible and could be discussed with the decision maker as long as the rule relates to the notion of similarity. Having $\beta_{k}\left(a_{D M}\right)$ 
defined allows us to bring forward the following working assumption:

Assumption 2 Let us consider $a_{i}$ and $a_{j}$ in $A$. If $a_{i} \in \beta_{k}\left(a_{j}\right)$, it is realistic to think that $a_{j}$ can improve to match the performance of $a_{i}$ on $g_{k}$, without significant degradation of performance on the other criteria.

For the sake of notation, let us consider any performance $x \in E$ such that there exists a criterion $g_{k}$ that identifies an observed alternative $\beta_{k}(x) \in A$. In this case, the performance of $x$ will evolve towards $x_{-k} \beta_{k}(x)$ (a performance improvement) which will be summarized as $\rho_{k}(x)$.

$$
\rho_{k}(x)=x_{-k} \beta_{k}(x)
$$

Step 4 - If only one criterion must be improved, one must be able to decide which one allows the most realistic improvement in terms of bottlenecks. We have seen that negative synergies are limited in our framework by using $a_{i}$ as a possible intermediate benchmark to improve $a_{D M}$ on a criterion $g_{k}$ if $\Delta_{k}\left(a_{D M}, a_{i}\right)>0$. We have mentioned that positive synergies will be accounted for as a post-analysis when paths of improvement are already generated. To be more precise, when two paths are optimal and only differ in the order of improvement of two consecutive steps inspired from the same observed alternative and involving two different criteria $g_{k}$ and $g_{j}$, there is no reason to choose to improve $g_{k}$ before $g_{j}$ nor the contrary. In such case, the question whether to consider these two steps as positive synergies will be studied with the decision maker. If applicable, these two consecutive steps could be seen as a joint step consisting of improving only one of the criteria but resulting in the increase of both of them by positive synergy. Bottlenecks must still be considered. We suppose that if bottlenecks exist, they might have been experienced by others and ultimately have been avoided. For this reason, we advise that $x$ should evolve as the majority of the better observed alternatives. In order to do so, we propose the following assumption:

Assumption 3 Let us consider the performance $p^{q} \in p$. The larger the cardinality of $\Lambda_{k}\left(p^{q}\right)$, the fewer the chances to encounter a bottleneck while improving the performance on criterion $g_{k}$.

This assumption suggests following the path that always ensures the highest number of future opportunities for improvement (which is supported by observed alternatives). This proposition results from a simple observation: without a bottleneck (or a negative synergy), an observed alternative can perform less well than it does on any single criterion without changing its performance on any other criterion. As a simplistic example, it is well known that in the retail business, a company needs volume to be able to increase its net benefit. If a retail company has enough volume, it can decide to reduce its net benefit or to keep it as high 
as possible (if one can do more, one can do less). However, if a retail company is expanding and does not yet have a large volume of sales, it cannot increase its net benefit to match that of its competitors with larger sales volumes. This proves to be a bottleneck.

When a bottleneck exists, it creates empty zones in the performance space which cannot be reached (supported) by any observed alternative. In opposition to that, the zones in the performance space that are reachable (not hindered by bottlenecks) are naturally more dense in terms of observed performance. Therefore, choosing to improve the criterion $g_{k}$ with the largest $\# \Lambda_{k}\left(p^{q}\right)$ as the next step starting from $p^{q}$, is a way of taking into account this observation and of building paths of improvement in dense zones of the performance space. If many observed alternatives exist that can act as future opportunities of improvement on a criterion $g_{k}$ and very few on a criterion $g_{h}$, we suggest improving on $g_{k}$ for its larger density of observed performances along the direction of the single performance improvement. It is worth noting that this rule naturally adds a positive impact regarding the robustness of the path of improvement as it suggests always keeping as many possible future opportunities of improvement. Let us define our final assumption based on this rule:

Assumption 4 Let us consider the performance $p^{q} \in p$. The focused improvement facing the fewest risk of barriers to improvement is $p^{q+1}=\rho_{h}\left(p^{q}\right)$ such that

$$
h=\underset{k=1 \cdots n}{\arg \max } \# \Lambda_{k}\left(p^{q}\right)
$$

If several $g_{h}$ with the same maximum value of $\# \Lambda_{h}\left(p^{q}\right)$ exist, then any of these directions are as good as another in terms of barriers to improvement. This section has presented the theoretical basis of this paper. Section 4 will describe the construction of the graph that will help generate several improvement scenarios.

\section{Definition of a graph}

Steps 1 to 4 enable to build a directed graph $\Gamma=(N, \Sigma)$ where $N \subseteq E$ is a set of vertices and $\Sigma \subseteq N^{2}$ is a set of edges. The starting vertex of $\Gamma$ is $g\left(a_{D M}\right)$. It is built repeatedly as follows:

1. Initiate the set of vertices as follows: $N=\left\{g\left(a_{D M}\right)\right\}$. Also initiate a set of new vertices: $H=$ 
$\left\{g\left(a_{D M}\right)\right\}$

2. For each vertex $\nu \in H$ :

(a) For each criterion $g_{k}$ such that $\Lambda_{k}(\nu) \neq \emptyset$ : If $\rho_{k}(\nu) \notin N$ add $\rho_{k}(\nu)$ to $H$, add $\rho_{k}(\nu)$ to $N$. Add the edge $\left(\nu, \rho_{k}(\nu)\right)$ to $\Sigma$ (for all $\left.g_{k} \in G\right)$;

(b) Remove $\nu$ from $H$;

3. Repeat 2 until $H=\emptyset$.

It is obvious that there cannot be more than $n$ outgoing edges from a given vertex in $N$. Each edge $\epsilon \in \Sigma$ such that $\epsilon=\left(\nu, \rho_{k}(\nu)\right)$ with $\nu \in N$ will be characterized by three indicators related to bottlenecks, operational changes and rank/class increase of $\epsilon$. We define an operational change, in a path of improvement $p$, as a step $p^{q}$ that improves a criterion $g_{k}$ different from the criterion improved at step $p^{q-1}$ and which is based on a different observed alternative. Therefore, it cannot be solely defined on the basis of an edge $\epsilon$ : it depends by nature on two consecutive edges.

In order to properly model the operational changes, we define a meta-graph $\Gamma^{*}=\left(N^{*}, \Sigma^{*}\right)$ where:

- $N^{*}=\Sigma \cup\left\{\left(g\left(a_{D M}\right), g\left(a_{D M}\right)\right)\right\}$ is the set of vertices;

- $\Sigma^{*} \subseteq N^{* 2}$ is the set of edges, to which costs are associated and will be defined below in this section.

Imposing that $N^{*}=\Sigma \cup\left\{\left(g\left(a_{D M}\right), g\left(a_{D M}\right)\right)\right\}$ means that all the edges from $\Gamma$ become vertices in $\Gamma^{*}$. In addition to that, an idle vertex, $\left\{\left(g\left(a_{D M}\right), g\left(a_{D M}\right)\right)\right\}$ (which will be used as a starting vertex for $\Gamma^{*}$ ) is added to $N^{*}$.

Vertices of $\Gamma^{*}$ - Let us consider a criterion $g_{k}$. If an edge $\epsilon^{*}=\left(\nu, \rho_{k}(\nu)\right) \in \Sigma$ exists, then $\epsilon^{*} \in N^{*}$.

Edges of $\Gamma^{*}$ - Let us assume that $\nu^{\prime} \in N$ exists such that $\nu^{\prime}=\rho_{k}(\nu)$ and that $g_{h}$ exists such that $\epsilon^{*^{\prime}}=\left(\nu^{\prime}, \rho_{h}\left(\nu^{\prime}\right)\right) \in \Sigma$. Then, $\left(\epsilon^{*}, \epsilon^{*^{\prime}}\right)=\left(\left(\nu, \rho_{k}(\nu)\right),\left(\nu^{\prime}, \rho_{h}\left(\nu^{\prime}\right)\right)\right) \in \Sigma^{*}$.

Before defining each individual indicator, a function $\tau: E^{2} \rightarrow \mathcal{R}$ must be defined. It relates to the effort of execution of a step that consists of a single performance improvement from an initial performance $x \in E$ to a final performance $y \in E$. In an ideal situation, such functions should be built through a direct or indirect 
procedure with the decision maker. Otherwise, it is possible to use the difference of rank or level between $x$ to $y$ as a proxy indicator. Equation 3 provides one possible definition for $\tau$.

$$
\tau(x, y)= \begin{cases}R\left(x, A^{*}\right)-R\left(y, A^{*}\right) & \text { if } R \text { is defined for the preference model } M \\ C\left(x, A^{*}\right)-C\left(y, A^{*}\right) & \text { else }\end{cases}
$$

Obviously, these functions will only be applied to $x, y \in E$ such that $y S x$. Therefore, in this case, $\tau: E^{2} \rightarrow\{0,1, \cdots, m-1\}$ ( $m$ being the amount of observed alternatives). Indeed, $m-1$ is the maximal rank difference between two alternatives. We will define the three indicators (bottlenecks, operational changes and rank/level increase) from this point on as functions of $\tau$ defined in Equation 3 . Indeed, we suppose that it would be hard to obtain difficulty functions through a direct procedure and no adequate indirect elicitation procedure exists in the literature (to the best of our knowledge). It falls to future research to develop such a procedure. For the sake of simplicity, each edge $\left(\epsilon^{*}, \epsilon^{*^{\prime}}\right)=\left(\left(\nu, \rho_{k}(\nu)\right),\left(\nu^{\prime}, \rho_{h}\left(\nu^{\prime}\right)\right)\right) \in \Sigma^{*}$ will be noted $\left(\nu, \nu_{k}^{\prime}, \nu_{h}^{\prime \prime}\right)$, with $\nu_{k}^{\prime}=\rho_{k}(\nu)$ and $\nu_{h}^{\prime \prime}=\rho_{h}\left(\nu_{k}^{\prime}\right)$. Furthermore, it is important to note that using a difference of rank or level is from a mathematical point of view incorrect, as the difference of ordinal measures is mathematically meaningless. However, this allows to adopt a practical and decision-making-like approach which is often accepted in such a context. More generally, the sum of ordinal measures is mathematically incorrect because these measures are not built to be given more meaning than their ordinality. For a more mathematically correct but less discriminating approach, ordinal aggregation procedures could be used such as order statistics for example.

Bottlenecks - Let us characterize an edge $\left(\epsilon^{*}, \epsilon^{*^{\prime}}\right)$ in relation to bottlenecks. We suggest that the difficulty of execution associated to an improvement step should be minimized if it is not (one of) the most realistic step(s) with regard to bottlenecks. As a reminder, Assumption 3 proposes that the most realistic step to avoid a bottleneck is one whose direction of improvement ensures the largest amount of future improvement opportunities. The underlying idea is that if a step faces the risk of encountering a bottleneck mechanism, the efforts gathered for its implementation should be minimized as it could result in useless efforts. The possible losses of effort (through a bottleneck mechanism) will be denoted by $Z^{*}\left(\nu, \nu_{k}^{\prime}, \nu_{h}^{\prime \prime}\right)$ and are defined 
in Equation 4 .

$$
Z^{*}\left(\nu, \nu_{k}^{\prime}, \nu_{h}^{\prime \prime}\right)= \begin{cases}0 & \text { if } h=\arg \max _{\ell=1 \cdots n} \# \Lambda_{\ell}\left(\nu_{k}^{\prime}\right) \\ \tau\left(\nu_{k}^{\prime}, \nu_{h}^{\prime \prime}\right) & \text { else }\end{cases}
$$

Operational changes - Let us characterize an edge $\left(\epsilon^{*}, \epsilon^{*^{\prime}}\right)$ with regard to operational changes. We propose that the effort of execution of a step be minimized if it involves an operational change relative to the previous step. The reason is that an operational change can lead to a resistance to change which goes along with losses of resources or effort. Therefore, even though operational changes are necessary, the effort resulting from these should be minimized in order to reduce the possible loss of effort associated to them. The operational changes are denoted by $\Phi^{*}\left(\nu, \nu_{k}^{\prime}, \nu_{h}^{\prime \prime}\right)$ and defined in Equation 5 .

$$
\Phi^{*}\left(\nu, \nu_{k}^{\prime}, \nu_{h}^{\prime \prime}\right)= \begin{cases}0 & \text { if } \beta_{k}(\nu)=\beta_{h}\left(\nu_{k}^{\prime}\right) \text { or } k=h \\ \tau\left(\nu_{k}^{\prime}, \nu_{h}^{\prime \prime}\right) & \text { else }\end{cases}
$$

Rank/level increase - Let us define the rank or level increase related to an edge $\left(\epsilon^{*}, \epsilon^{*^{\prime}}\right)$ and denoted by $I^{*}\left(\nu, \nu_{k}^{\prime}, \nu_{h}^{\prime \prime}\right)$. It has to be maximized. It is a conflicting indicator with regard to $Z^{*}$ and $\Phi^{*}$ as it will attempt to generate scenarios of improvement with strong improvement of performance for $a_{D M}$. As $\tau$ has been defined in this paper as a function of the rank or level increase, one can define $I^{*}$ as in Equation 6 .

$$
I^{*}\left(\nu, \nu_{k}^{\prime}, \nu_{h}^{\prime \prime}\right)=\tau\left(\nu_{k}^{\prime}, \nu_{h}^{\prime \prime}\right)
$$

We can aggregate the three indicators into one final indicator, denoted by $\kappa^{*}\left(\nu, \nu_{k}^{\prime}, \nu_{h}^{\prime \prime}\right)$ maximized as:

$$
\kappa^{*}\left(\nu, \nu_{k}^{\prime}, \nu_{h}^{\prime \prime}\right)=I^{*}\left(\nu, \nu_{k}^{\prime}, \nu_{h}^{\prime \prime}\right)-\alpha_{\Phi} \cdot \Phi^{*}\left(\nu, \nu_{k}^{\prime}, \nu_{h}^{\prime \prime}\right)-\alpha_{Z} \cdot Z^{*}\left(\nu, \nu_{k}^{\prime}, \nu_{h}^{\prime \prime}\right)
$$

with $\alpha_{\Phi}, \alpha_{Z}$ being two penalty weights. The scale of these weights can differ depending on the scales of $Z^{*}, \Phi^{*}$ and $I^{*}$. For instance, if the three indicators are expressed as a function of $\tau$ defined in Equation 3 consequently they share the same scale and one can define $\alpha_{\Phi}, \alpha_{Z} \in[0,1]$ such that $\alpha_{\Phi}+\alpha_{Z} \in[0,1]$. This would prevent ever having a negative final indicator $\kappa^{*}$ and reduce the intensity of improvement in function of the penalty weights in case of bottleneck or operational change. In order to generate scenarios for improvement, one can simply generate multiple pairs of weights $\left(\alpha_{\Phi}, \alpha_{Z}\right)$ with the value of the sum $\alpha_{\Phi}+\alpha_{Z}$ that varies from its minimum value to its maximal value (for instance, from 0 to 1 ). We believe that it is not 
mandatory to find all efficient solutions to the problem. One can instead generate many efficient solutions and afterwards analyze them with the decision makers.

Each set of weights leads to a problem that can be seen as a shortest path problem. To find the solution of each single objective shortest path problem, we implement a Dijkstra algorithm. The graph being sparse $\left(\#\left(\Sigma^{*}\right)\right.$ equals at most $\left.n . \# N\right)$, if it is stored in the form of adjacency lists, the algorithm requires at worst a running time of $\left.\Theta\left(n . \# N^{*} \cdot \log \left(\# \Sigma^{*}\right)\right)\right)$ (Raman, 1997).

Then, one must define the global indicators related to the bottlenecks, the operational changes and the rank/level increase of a path $p$ in $\Gamma^{*}$, starting from $\nu_{1}=\left(g\left(a_{D M}\right), g\left(a_{D M}\right)\right)$ towards a given final vertex $\nu_{Q}$, as follows:

$$
\begin{aligned}
Z(p) & =\sum_{q=2}^{Q-1} Z^{*}\left(\nu_{q-1}, \nu_{q}, \nu_{q+1}\right) \\
\Phi(p) & =\sum_{q=2}^{Q-1} \Phi^{*}\left(\nu_{q-1}, \nu_{q}, \nu_{q+1}\right) \\
I(p) & =\sum_{q=2}^{Q-1} I^{*}\left(\nu_{q-1}, \nu_{q}, \nu_{q+1}\right)
\end{aligned}
$$

Finally, this procedure generates scenarios (or paths) of improvement and evaluates them. Let us remind the case in which we study the existence of positive synergies. When two consecutive single performance improvement steps of a path $p$ are based on the same observed alternative and could be inverted in the path without changing $Z(p)$ (inverting them does not change the risk of facing a bottleneck), then the question whether a positive synergy between these two criteria exists should be studied with the decision maker. Obviously, this principle applies to more than two consecutive steps. Moreover, when two consecutive steps are based on the same criterion to improve (but different observed alternatives), these steps can be seen as only one step with several sources of inspiration.

\section{Illustration on an MAVT model}

In order to illustrate the general framework with an additive Multi-Attribute Value Theory (MAVT) model, the following notation will be used: 
- $v_{j}: E_{j} \rightarrow[0,1]$, a non-decreasing and positive partial value function associated to criterion $g_{j}$;

- $w_{j} \in[0,1]$, a positive scaling factor that defines acceptable trade-offs for the decision maker such that

$$
\sum_{j=1}^{n} w_{j}=1
$$

- $V(x)=\sum_{j=1}^{n} w_{j} \cdot v_{j}\left(x_{j}\right)$ - the global value of $x \in E$.

It is worth noting that there is no restriction on the method used to obtain the scaling factors (such as the swing method used in Costa \& Vansnick (1999)) or the partial value functions. The latter can be obtained through an indirect rating procedure. For instance, the bisection or difference method von Winterfeldt \& Edwards, 1986). They can also be obtained by a direct rating through pairwise comparison and by solving a linear program as in the MACBETH methodology (Costa \& Vansnick, 1999) or in the Analytical Hierarchy Process (AHP) developed by Saaty et al. (2006). These two methods yield global values measured on an interval scale whose differences can be compared meaningfully (assuming a consistent construction of the latter partial values).

Step 1- This preference model allows to build a total order on any set of alternatives. Therefore, we suggest using $\tau(x, y)=R\left(x, A^{*}\right)-R\left(y, A^{*}\right)$. An important assumption used in this type of model is the mutual preferential independence between criteria (which allows the model to be additive). For the sake of clarity, if this assumption is validated, it does not interfere with our framework that states that structural dependencies between criteria exist. Preferential dependencies are one matter, structural dependencies are another.

Step 2- In an MAVT model, there are only two binary relations: strict preference or indifference. Therefore, any alternative $a_{i} \in A$ that has a higher value than $a_{D M}$ is considered to be better. One needs to define the function $\Delta_{k}\left(a_{D M}, a_{i}\right)$ that allows to limit the possible degradation of performances. We propose the following (assuming without loss of generality that all criteria must be maximized):

$$
\Delta_{k}\left(x, a_{i}\right)=\min \left(v_{k}\left(g_{k}\left(a_{i}\right)\right), b_{k}^{*}\right)-v_{k}\left(g_{k}(x)\right)-\left(\sum_{g_{j} \in G, g_{j} \neq g_{k}} \max \left(0, v_{j}\left(g_{j}(x)\right)-\max \left(\left(v_{j}\left(g_{j}\left(a_{i}\right)\right), b_{j *}\right)\right)\right)\right.
$$

If $\Delta_{k}\left(x, a_{i}\right) \geq 0$, it ensures improving only criterion $g_{k}$ as $a_{i}$ (to a maximum performance of $b_{k}^{*}$ ), even if there is a degradation of performances on other criteria $g_{j}$ (limited to a minimum performance of $b_{j *}$ ), such 


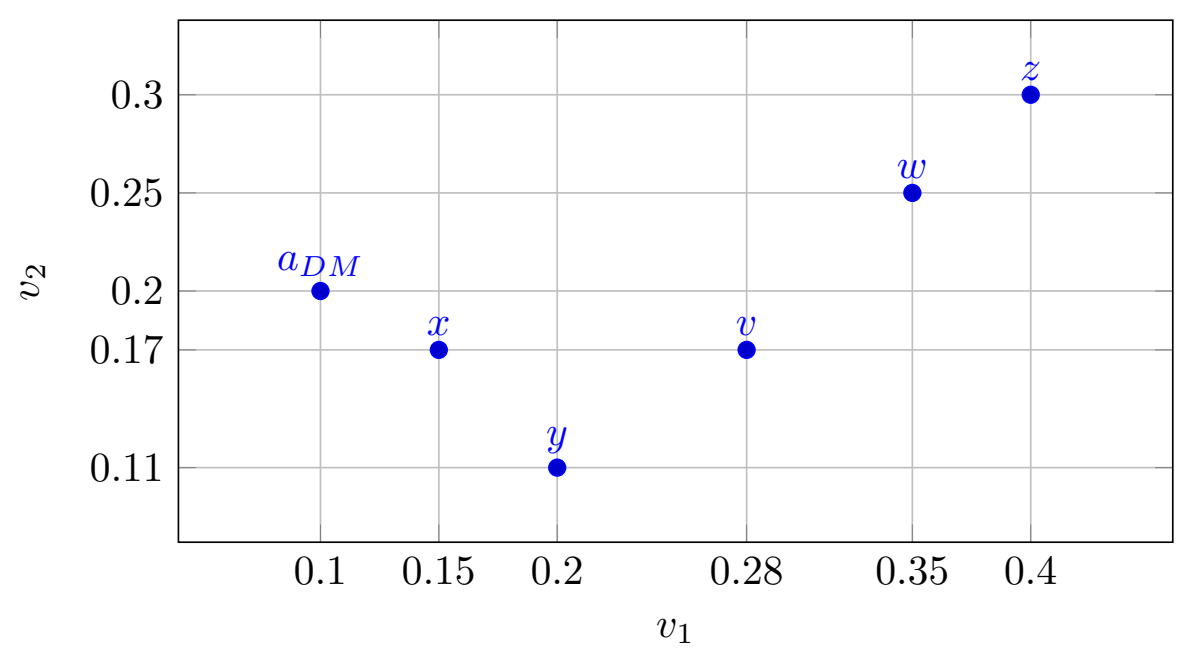

Figure 1: 6 observed alternatives represented in the value functions space

that the evolution of performances of $x$ will still be at least as good as before. Note that this function is a first proposition and could also be discussed with the decision maker.

Step 3- We suggest selecting $\beta_{k}(x)$ (for any performance $x$ ) by using the lexicographic rule proposed in Appendix A. This rule would be sufficient to ensure a unique $\beta_{k}(x)$ on any criterion $g_{k}$ if all pairs of observed alternatives perform differently on at least one criterion. For this reason, we will not develop it further. The rest of the operations and the creation of the graph result from these methodological choices. Let us now illustrate how we suggest building each $\Lambda_{k}$.

Example 1 Let us consider 5 observed alternatives ( $a_{D M}$ not included) whose performances denoted by $v$, $w, x, y, z$ are presented in Figure 1. Let us assume for the sake of simplicity that there is no limitation on the worst and best performance that $a_{D M}$ can reach on each criterion.

For the sake of notation, these alternatives are denoted by the same notation as their performance, i.e., $v, w$, $x, y$ and $z$. Similarly, the performance of $a_{D M}$ is presented in Figure 1 and denoted for the sake of notation as $a_{D M}$. Moreover, in order to distinguish the general theoretical notions from the application to an MAVT model, we will substitute, in this example, the notation $\Lambda_{k}\left(a_{D M}\right)$ for $L_{k}\left(a_{D M}\right)$.

One can easily see that the cardinality of $L_{1}\left(a_{D M}\right)$ is greater than that of $L_{2}\left(a_{D M}\right)$. Table 1 shows the values of $\Delta_{1}\left(a_{D M},.\right)$ and $\Delta_{2}\left(a_{D M},.\right)$ for each other observed alternative. Only the positive values of $\Delta_{1}\left(a_{D M},.\right)$ 
and $\Delta_{2}\left(a_{D M},.\right)$ will include the corresponding observed alternative in $L_{1}\left(a_{D M}\right)$ and $L_{2}\left(a_{D M}\right)$ (respectively).

\begin{tabular}{l|lllll}
\hline & $\mathrm{x}$ & $\mathrm{y}$ & $\mathrm{v}$ & $\mathrm{w}$ & $\mathrm{z}$ \\
\hline$\Delta_{1}\left(a_{D M},.\right)$ & 0.02 & 0.01 & 0.15 & 0.25 & 0.3 \\
$\Delta_{2}\left(a_{D M},.\right)$ & -0.03 & -0.09 & -0.03 & 0.05 & 0.1 \\
\hline
\end{tabular}

Table 1: Possible improvement target on criterion $g_{k}$ for $a_{D M}\left(\right.$ if $\Delta_{k}\left(a_{D M},.\right)>0$ )

As a consequence, we have $L_{1}\left(a_{D M}\right)$ and $L_{2}\left(a_{D M}\right)$ as in Equation 12 .

$$
\left\{\begin{array}{l}
L_{1}\left(a_{D M}\right)=\{x, y, v, w, z\} \\
L_{2}\left(a_{D M}\right)=\{w, z\}
\end{array}\right.
$$

Assumption 2 would suggest that two realistic improvements with the lowest degradation of performance are either $b_{1}\left(a_{D M}\right)=x$ or $b_{2}\left(a_{D M}\right)=w$. Assumption 3 suggests that the current most realistic improvement with regard to bottlenecks is to match the performance of $x$ on the first criterion. Our idea to avoid bottlenecks is illustrated in this first example: $a_{D M}$ improves by ensuring as many future opportunities of improvement as possible. One can see that it also leads to improving in a similar way to the majority of the observed alternatives. Indeed, choosing to improve the second criterion to match the performance of $w_{2}$ would lead to improving $a_{D M}$ in a more dissimilar way than $x, y$, v. Now, let us carry out the same exercise for $x$. Table 2 shows the $\Delta_{k}(x,$.$) values for k=1,2$.

\begin{tabular}{l|llll}
\hline & $\mathrm{y}$ & $\mathrm{v}$ & $\mathrm{w}$ & $\mathrm{z}$ \\
\hline$\Delta_{1}(x,)$. & -0.01 & 0.13 & 0.2 & 0.25 \\
$\Delta_{2}(x,)$. & -0.06 & 0 & 0.08 & 0.13 \\
\hline
\end{tabular}

Table 2: $\Delta_{k}$ values for $x$

This leads to define $L_{1}(x)$ and $L_{2}(x)$ as in Equation 13 .

$$
\left\{\begin{array}{l}
L_{1}(x)=\{v, w, z\} \\
L_{2}(x)=\{w, z\}
\end{array}\right.
$$

Based on Assumptions 2 and 3. the most realistic step to avoid bottlenecks and limit the negative synergies, is to reach the performance of $v$ on the first criterion. Obviously, it is possible that $x$ suffers a slight degradation of performance on the second criterion, but if it does, it will not be as significant as the increase in value 
on the first criterion. However, increasing the performance of $x$ on the second criterion to match that of $w$ is more likely to bring about a bottleneck, as suggested in Assumption 3. Finally, by repeating the same procedure for $v$, our assumptions state that improving the first criterion or the second is equally realistic. In this case the step of improvement suggested would be to improve as $w$ on both criteria. Hence, positive synergies could be studied for this step.

\section{Case study: University rankings}

This section proposes an illustrative case-study on which we have worked, as academic members of the Université libre de Bruxelles (ULB), in order to generate strategic plans to improve the ULB according to the Shanghai Academic Ranking of World Universities 2020 (ARWU20). The idea was to generate scenarios of improvement in the formal representation and then investigate on several steps of improvement in order to propose real life actions that could match these scenarios of improvement. It is worth noting that our conclusions have been appreciated by other members of the university but should only be taken as proof of concept. This is not (necessarily) the strategic plan that the ULB will follow.

Several rankings that assess a university's quality exist. The ARWU20 has been chosen (although it has faced much criticism (Billaut et al. 2010) ) due to its worldwide renowned status and its more quantitative criteria in comparison to the QS ranking or the Times Higher Education (THE) ranking. Indeed both rankings apply surveys which account for a large part of the evaluation of some criteria and cannot be verified, risking therefore more arbitrariness.

The universities considered are either from Belgium, France, the Netherlands or Germany. They must be of a similar or larger size than the ULB (greater than 12000 students using the size classification of the QS ranking). They must be composed of all faculties (except medical school, which is not mandatory).

The goal of this study was to find ways or conditions to reach the comparable Western-European top-ofthe-class universities. For this reason, the evaluations provided by ARWU20 were used to establish partial values through a simple bisection method (von Winterfeldt \& Edwards, 1986) resorting to only 3 middle points and 2 extreme values (the lowest yielding a value of 0 and the largest a value of 1 ). Linearity was then assumed between each consecutive middle value. The weights used in the ARWU20 were then used as 
scaling factors for the aggregation of the partial values.

The six criteria and their respective (normalized) scaling factors are the following ones (Billaut et al. 2010):

1. ALUMNI (10\%): Former students having been awarded (medals or prizes);

2. AWARDS (20\%): Academic members of the staff having been awarded;

3. HiCi (20\%): Number of highly cited members of staff;

4. N\&S (20\%): Number of citations in Nature and Science;

5. PUB (20\%): Volume of published papers (from Clarivate database);

6. PCP (10\%): Mean score of the 5 latter criteria divided by the total amount of students (it can be interpreted as an efficiency criterion).

The partial values of each university multiplied by the scaling factor of each criterion are provided in Table

3. The only restrictions on performance change for the ULB were to prevent any degradation and allow very

\begin{tabular}{llllllll}
\hline Universities & Abbrev. & Alumni & Award & HiCi & N\&S & PUB & PCP \\
\hline Ghent University & BEL_Ghent & 0 & 0,0258 & 0,0672 & 0,035 & 0,1212 & 0,0341 \\
KU Leuven & BEL_KU & 0 & 0 & 0,0542 & 0,0484 & 0,1218 & 0,0296 \\
Université Libre de Bruxelles & BEL_ULB & 0,0188 & 0,0514 & 0,0198 & 0,034 & 0,0678 & 0,0325 \\
Paris Saclay & FRA_Sacl & 0,0284 & 0,1492 & 0,0852 & 0,0596 & 0,132 & 0,0318 \\
Sorbonne University & FRA_Sorbonne & 0,0345 & 0,053 & 0,0464 & 0,0576 & 0,128 & 0,0261 \\
University of Bonn & GER_Bonn & 0,023 & 0,069 & 0,0396 & 0,033 & 0,0766 & 0,0236 \\
University of Freiburg & GER_Frei & 0,0217 & 0,0364 & 0,0314 & 0,0392 & 0,0802 & 0,0209 \\
University of Göttingen & GER_gott & 0,0287 & 0,0288 & 0,0342 & 0,0424 & 0,078 & 0,0212 \\
Heidelberg University & GER_Heidelberg & 0,0188 & 0,049 & 0,0442 & 0,0562 & 0,1068 & 0,0276 \\
LMU Munich & GER_LMU & 0,026 & 0,037 & 0,0524 & 0,0616 & 0,1106 & 0,0289 \\
University of Groningen & NET_GRON & 0,0133 & 0,0492 & 0,042 & 0,0348 & 0,1098 & 0,0317 \\
Leiden University & NET_Leiden & 0,0154 & 0,0182 & 0,056 & 0,0426 & 0,108 & 0,0313 \\
Radboud University Nijmegen & NET_Radboud & 0,0163 & 0 & 0,0504 & 0,0368 & 0,1068 & 0,0371 \\
University of Amsterdam & NET_University & 0 & 0 & 0,0504 & 0,0382 & 0,1134 & 0,0298 \\
Utrecht University & NET_Utrecht & 0,0217 & 0,0386 & 0,0642 & 0,0468 & 0,1096 & 0,0288 \\
Vrije Universiteit Amsterdam & NET_VrijAMS & 0 & 0 & 0,0578 & 0,0254 & 0,1074 & 0,0328 \\
\hline
\end{tabular}

Table 3: Partial values multiplied by each criterion's scaling factor for the ARWU20

few improvement on the Alumni and Award criteria as the ULB has practically no control over it, given that the medals and prizes it has received cannot be withdrawn. Therefore $b_{\text {Alumni* }}=0.0188, b_{\text {Alumni }}^{*}=0.02$ and $b_{\text {Award* }}=0.0514, b_{\text {Award }}^{*}=0.055$ (expressed in scaled partial value). Regarding the penalty weights used to aggregate the indicators weighting the graph, a set of 54 pairs were used to generate scenarios of improvement, covering the whole area of possible weight values such that $0<\alpha_{\Phi}+\alpha_{Z} \leq 1$ and $0<\alpha_{\Phi}, \alpha_{Z}<1$. 
Finally, it was decided that in the definition of $\Lambda_{k}$ (the set of universities that can be used as sources of inspiration at each step in order to improve on a criterion $g_{k}$ ), another constraint would be added: the improvement should at least induce a rank increase equal to 1 . The minimum number of rank(s) expected to be increased at each step can also be typed into the software when the user decides to generate the paths of improvements. In total, 5 paths of improvement have been generated.

\begin{tabular}{|c|c|c|c|c|c|c|}
\hline Path & step $1 / 5$ & step $2 / 6$ & step $3 / 7$ & step $4 / 8$ & $Z(p)$ & $\Phi(p)$ \\
\hline$p_{1}$ & $\begin{array}{l}\text { GER_gott - HiCi }+(3) \\
\text { GER_Heidelberg - HiCi }+(2)\end{array}$ & $\begin{array}{l}\text { NET_Utrecht - N\&S (+1!B!) } \\
\text { GER_LMU - HiCi +(1) }\end{array}$ & $\begin{array}{l}\text { GER_Heidelberg - N\&S (+1!B!) } \\
\text { FRA_Sacl - HiCi + (1) }\end{array}$ & GER_Heidelberg - PUB +(4) & 2 & 4 \\
\hline$p_{2}$ & $\begin{array}{l}\text { GER_gott - HiCi + }(3) \\
\text { BEL_Ghent - PUB }(+1 ! B !)\end{array}$ & $\begin{array}{l}\text { GER_Heidelberg - HiCi +(1) } \\
\text { FRA_Sacl - PUB }(+2 ! B !)\end{array}$ & $\begin{array}{l}\text { NET_Radboud - HiCi }(+1 ! \mathrm{B} !) \\
\text { FRA_Sacl - HiCi }(+1 ! \mathrm{B} !)\end{array}$ & NET_Radboud - PUB + (4) & 5 & 3 \\
\hline$p_{3}$ & $\begin{array}{l}\text { GER_gott - HiCi + (3) } \\
\text { NET_Utrecht - N\&S +(1) }\end{array}$ & $\begin{array}{l}\text { GER_Heidelberg - HiCi + (1) } \\
\text { BEL_KU - HiCi + (1) }\end{array}$ & $\begin{array}{l}\text { NET_Radboud - HiCi }(+1 \text { !B!) } \\
\text { BEL_Ghent - PUB + }(1)\end{array}$ & $\begin{array}{l}\text { NET_Radboud - PUB +(4) } \\
\text { FRA_Sacl - HiCi + (1) }\end{array}$ & 1 & 6 \\
\hline$p_{4}$ & $\begin{array}{l}\text { GER_gott - HiCi +(3) } \\
\text { NET_Utrecht - N\&S +(1) }\end{array}$ & $\begin{array}{l}\text { GER_Heidelberg - HiCi + (1) } \\
\text { GER_LMU - PUB + }+(1)\end{array}$ & $\begin{array}{l}\text { NET_Radboud - HiCi }(+1 ! \mathrm{B} !) \\
\text { NET_Utrecht - HiCi + (1) }\end{array}$ & $\begin{array}{l}\text { NET_Radboud - PUB +(4) } \\
\text { FRA_Sacl - PUB }+(1)\end{array}$ & 1 & 6 \\
\hline$p_{5}$ & $\begin{array}{l}\text { GER_gott - HiCi + (3) } \\
\text { GER_LMU - HiCi +(1) }\end{array}$ & $\begin{array}{l}\text { GER_Heidelberg - HiCi +(1) } \\
\text { FRA_Sacl - HiCi +(1) }\end{array}$ & NET_Radboud - PUB + (5) & GER_Heidelberg - N\&S +(2) & 0 & 11 \\
\hline
\end{tabular}

Table 4: The five optimal paths of improvement for the ULB

Table 4 shows that in terms of the number of possible bottlenecks and operational changes, all paths are considerably distinct. Each step should be read as follows: University - criterion to improve +(rank's increase) and if the step risks facing a bottleneck, a !B! is indicated next to the rank's increase. After much thought, it was suggested that $\Phi(p)$ should be larger than $Z(p)$ because operational changes are mandatory in order to improve, and as a university it would be better to avoid wasting resources in bottlenecks. An analysis on the optimality of each path for each pair of penalty weights was conducted. It appears that $p_{1}$ is optimal for $56 \%$ of the pairs of penalty weights whereas $p_{3}, p_{4}$ and $p_{5}$ are optimal for around $11 \%$. Figures 2, 3 show the areas (in green) for which $p_{1}$ and $p_{3}$ are optimal. Figure 4 provides a visualization of $p_{1}$. Both figures are produced at will for each path in the software. The title of Figure 4 provides the percentages $(56 \%)$ and the centroid $\left(\alpha_{Z}=0.36, \alpha_{\Phi}=0.36\right)$ of the pairs of penalty weights for which the path is optimal. This allows to already provide an idea of what Figure 2 would show.

Let us analyze the skeleton of $p_{1}$ as it is the most robust in terms of penalty weights. A first step would be to improve the number of highly cited academic members as is the case for the University of Gottingen (GER_Gott). This university has 5 members who fit this category: Lutz Ackermann, professor in chemistry, Teja Tscharntke, professor in agroecology, Tobias Plieninger, professor in agricultural science, Ivo Feußner, 


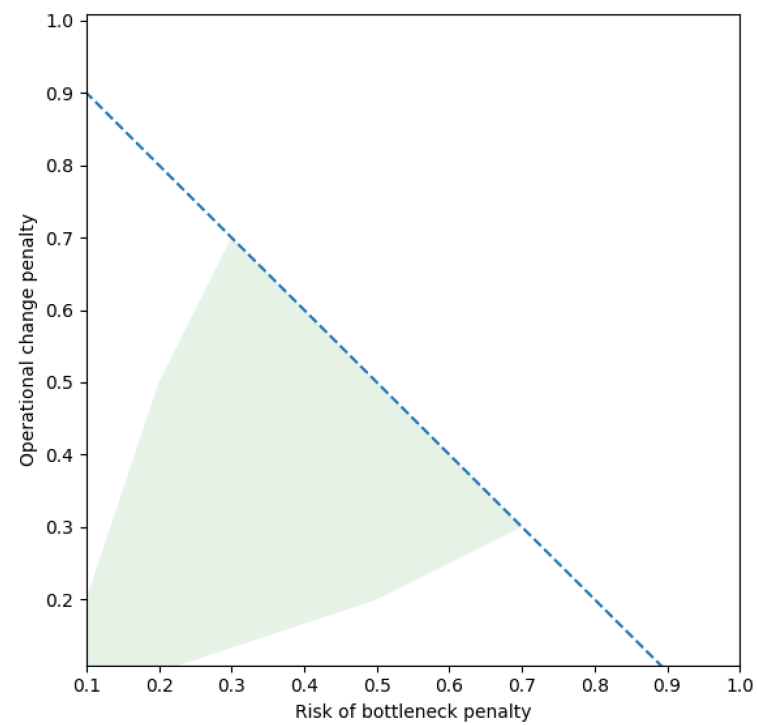

Figure 2: Software interface: Penalty weights supporting the optimality of $p_{1}$

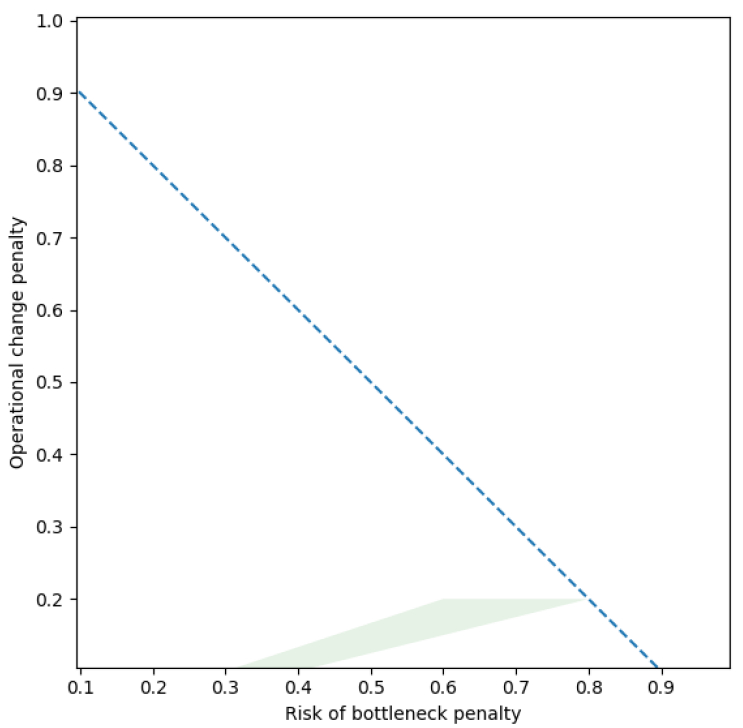

Figure 3: Software interface: Penalty weights supporting the optimality of $p_{2}$

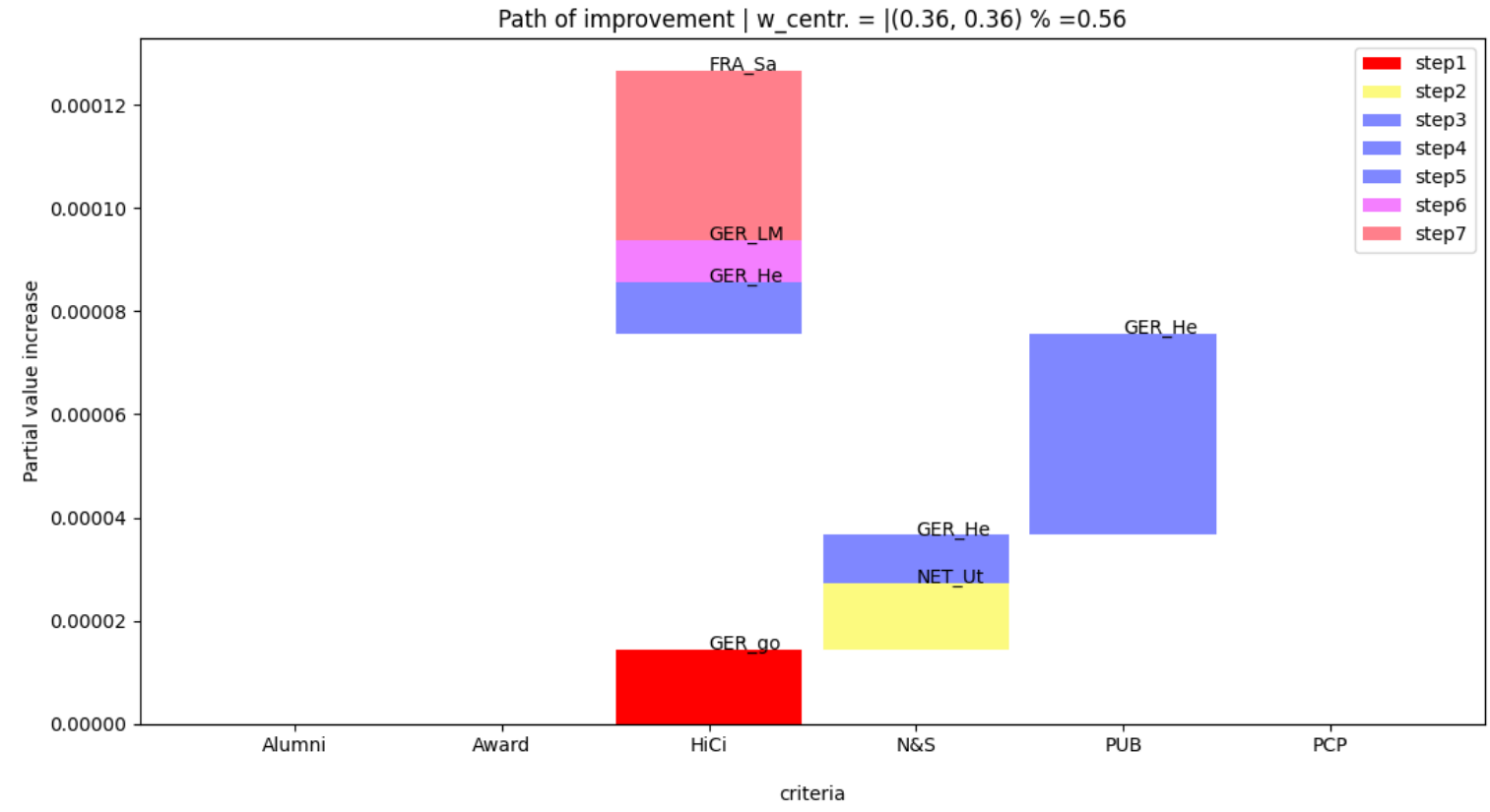

Figure 4: Software interface: $p_{1}$ decomposed step by step

professor in biochemistry and Holger Kreft, researcher in biodiversity. It is interesting to note that these researchers work in similar fields. Let us verify if it can also be applied for the University of Heidelberg which is also part of the recommendation. It can be read on the university's website that there are 13 
researchers among the highly cited members including Prof. Dr Ralf Bartenschlager (microbiology), Prof. Dr A. Stephen K. Hashmi (chemistry), Prof. Dr Hugo A. Katus (medicine), Prof. Dr Andreas von Deimling (neuroscience and behaviour), Prof. Dr Markus W. Büchler (medicine), Prof. Dr Hartmut Goldschmidt (medicine), Prof. Dr Rolf-Detlef Treede (neuroscience and behaviour). The others are considered cross-field (meaning that they have published in different fields). It is interesting to remark that again, there seems to be a dominant field of expertise for this university: medicine. When looking at the ULB, its dominant field of expertise could be physical science, for which it has recently received a Nobel Prize. There is not, seemingly, another field of expertise according to the rankings by faculty also proposed by ARWU on their website. One strategic action could be to develop this field in particular (by providing more money to invest in related research projects or in European/ international research networks, and by taking advantage of the reputation that the ULB has acquired thanks to the Nobel Prize, in order to be part of these networks). Another strategic action could be to analyze which faculties and fields are the most promising and consequently invest academic research funds accordingly. This has been done for instance in Heidelberg. Resources are allocated according to the research results (as is claimed on their website). This is not the case for the ULB, which regards freedom of research as fundamental, even for fields that are less appealing. It is worth noting that a recommendation in the formal representation can yield different strategic actions.

A second recommendation for the ULB in $p_{1}$ is to take Heidelberg University as a source of inspiration in order to improve the number of papers published in Nature and Science. This depends obviously on the field of expertise of the university. If one supposes that more money is spent on promising scientific research projects, as recommended to improve the HiCi criterion, N\&S could already easily be improved if the ULB pushes its researchers to submit their paper to Nature and Science before any other journal. To the best of our knowledge, this is not commonly practiced at the ULB. It is then recommended to increase the volume of published papers (PUB) similarly to Heidelberg University. Logically, if resource allocations are distributed according to publication results, researchers will be willing to increase publishing. Yet there are additional ways of improving the volume of research. We have investigated the research strategy of Heidelberg University. It appears that from 2015 to 2016, the university increased its industry income (as was declared for the Times Higher Education 2015 ranking), which led to a significant rise in research volume in 2016 (this information is also available on the THE website). This illustrates another strategic action: accepting and promoting further research collaborations with the industry. This allows to bring in money and more 
research topics which are likely to be used, and as a result, inevitably increase the volume of research. Again, the ULB has a reputation of promoting freedom of research and of drastically limiting its income from the industry. However, it was suggested by members of staff working on the ULB strategy that this strategic action could more likely be accepted with the support of a study such as ours as it shows potential positive outcomes in the formal representation as well as in reality. Moreover, Heidelberg University invests largely in international networks of research, which allows its members to participate in cross-field and cross-university projects, thus providing them with research opportunities and therefore with more chances to publish. This strategy is in accordance with one of the previously mentioned strategy to increase the highly cited academic members. A wider network could result in a greater reputation likely to improve the university's attractiveness, therefore encouraging more individuals to be part of the staff and consequently raise the probability to increase the number of highly cited members.

Finally, the number of highly cited members could still be improved. This could be a consequence of the past strategic actions as explained above (and thus be considered as a positive synergy). Yet, let us focus on the last step: Paris Saclay (FRA_Sa). This case is highly interesting and different, as Paris Saclay, previously known as the University Paris Sud before 2019, merged with well reputed schools in 2020, allowing it to climb quickly in the ARWU20 ranking. Indeed, it gathered under one institution all the medals, prizes and publications from the merged institutions. This strategic action undoubtedly allows to improve in the ranking. The difficulty in the case of the ULB is that, unlike Paris Sud, it is already a leading French-speaking university in Belgium. Moreover, the Dutch-speaking universities currently have a very different operational system, making the idea of a merge far more difficult. However, if the ULB came to play the game of industry income and strove to enter more international research networks it could match the interests of the KU Leuven or of Ghent University, these being the two leading Flemish universities in Belgium. A last major difficulty with this strategic action is that a merge is political as these are state universities. Therefore there are many conditions that must be fulfilled for the merge to be legal.

To conclude, this path of improvement $\left(p_{1}\right)$ in the formal representation matches a sequence of strategic actions. The latter should be evaluated more thoroughly in order to assess the cost (in euros, but also other costs, such as the social cost of pressuring researchers to publish, for instance). This procedure could be considered for each path of improvement in order to generate different sequences of strategic actions that 
could ultimately be compared to one another. The results of this study were presented to the manager of Statistics and Prospective Studies Office at ULB whose opinion follows: As part of the direction for strategic support of the ULB I can attest to the usefulness of such a study. Given that universities are forced to operate in drastically different conditions between neighbouring countries or even regions, the task of comparing institutions and their performances becomes a complex one. The different strategies proposed not only give us some insight on the potential improvements we could make but also highlight some weaknesses that we have when compared to others of a similar size. Obviously some actions can be hard to implement when they go against the stance of our institution or when a local legislation makes them difficult if not impossible. However, in an ever changing context where several universities are experiencing a period of growth, it is that much more important to realise which criteria are important to improve or maintain. As a matter of fact, a recurrent yearly increase in students comes with a tighter management of our resources and knowing which aspects to prioritise and on which not to compromise is valuable information.

\section{Discussion and Limitations}

Our framework faces a few limitations. Firstly, many methodological choices must be made which are not unique. For instance, when adapting it to an MAVT model, Assumption 2 uses a very limited extension of the dominance relation (Equation 11) to determine the possible steps of focused improvement. The advantage of a set of performances $x$ towards other performances $y$ must be larger on one criterion $g_{k}$ than the disadvantages on the rest of the criteria in order to state as realistic that an alternative performing like $y$ could reach $g_{k}\left(x_{k}\right)$, without a significant degradation of performances on the other criteria. If a large number of criteria exists, this definition could become too restrictive to propose realistic recommendations or any recommendation at all.

Secondly, in order to find the optimal solutions of the graph generated by our framework, we need to sum up the rank increases, which is an ordinal information. It imposes implicitly very strict assumptions regarding the intensity of each difference of rank. For instance, it imposes that the intensity of improvement of one rank is the same starting from any initial rank. Modelling the intensity of improvement as well as the difficulty of improvement of any step is a very complex task which must still be studied. As explained, this aspect is not developed in this paper but will be part of future research to study how to obtain a global difficulty function based on partial difficulty functions obtained in the same way partial value functions are built (if it 
makes sense to define such functions). It is not an easy task, as preferences and difficulties are of a different nature. For instance, there are often structural dependencies in a problem where preferential independence or its relaxed form, weak separability, could be accepted allowing for a summation of the partial values but perhaps not for the partial difficulties. A fitted elicitation method and aggregation operator must be built accordingly. However, as explained previously, in many cases using a proxy is sufficient as the scenarios of improvement will only be the basis to generate real strategic actions.

Thirdly, an apparent strong hypothesis is made. All the observed alternatives remain in an idling mode while the studied alternative improves. This is obviously not realistic. Nonetheless, this is a way to understand how it is possible for an alternative to improve at a given moment. Scenarios of improvement could be re-generated a month or a year later in order to reassess and adapt the strategy. Hence, it is important to develop the robustness concern in the generated scenarios to point out sensitive data.

About robustness, it is implicitly assumed in this paper that the additive value model used to evaluate the alternatives is unique. This is generally not true and one model is selected arbitrarily. Indeed, one usually limits the amount of questions during the elicitation of the marginal additive value functions in order to reduce the cognitive effort of the decision maker, which allows for an (assumed low) indetermination. One could study the possible and necessary optimal paths in the same vein as Kadziński et al. (2016) who study the necessary and possible binary relation between each pair of alternatives. Moreover, other robustness analyses could be carried out, such as withdrawing one or several observed alternatives and studying the deviation on the generated paths, or using a metric, like the Hausdorff distance, between each generated path in order to evaluate their similarity, etc.

Finally, a limitation of our model is the fact that the possible degradation of performances is not taken into account by the paths of improvement. It is only limited by Assumption 2. This is a difficult matter that has never been, to the best of our knowledge, properly tackled in the literature but should be the subject of a serious study in the future, as previously explained. 


\section{Conclusion}

Let us summarize the important ideas of this article. A new framework is proposed aiming at generating scenarios of improvement. It is based on novel assumptions with regard to the literature on stepwise benchmarking. It takes into account practical considerations such as operational changes and the risk of bottlenecks while generating the scenarios. Moreover, it acknowledges the fact that benchmarks are often based on abstract formal representations of real goals. This means that a stepwise benchmarking method should help in such a case to study observed alternatives and understand how/why they perform better in the formal representation. For these practical considerations, we propose that each step of improvement be focused on one criterion. As a consequence, we allow a scenario of improvement to be built with fictitious alternatives that are supported by observed alternatives. Moreover, this framework is defined to be adaptable to different MCDA models. It is not restricted to a particular model. For illustrative purposes, we have adapted it to a classical MAVT model. Further research could be dedicated to its adaptation to an outranking preference model such as ELECTRE. Indeed, in the case of methods with cyclic outranking relations, additional hypotheses should be made, especially regarding the rank/level increase. Other models like PROMETHEE (Brans \& De Smet, 2016) could be more easily integrated.

A weighted graph with three objectives is built, whose optimal solutions are computed with a Dijkstra shortest path algorithm by aggregating the three objectives for several representative set of penalty weights. One could also use a bi-objective Dijkstra algorithm to compute the Pareto Optimal frontier of the graph, with one objective being the rank or level increase, and the second being the aggregation of the effort of execution related to operational changes and to possible bottlenecks. Indeed, if one is able to build difficulty functions that are independent from the rank or level increase, the two objectives would not share the same scale anymore. Hence, aggregating the three objectives would not be meaningful.

\section{Acknowledgements}

We would like to thank Karim Lidouh, head of the Statistics and Prospective Studies Office at ULB for his precious comments on our case-study and the two anonymous referees for their insightful comments helping improving this article. 


\section{A Appendix: Selection of the most similar alternative in $\Lambda_{k}$}

When several candidates for $\beta_{k}\left(a_{D M}\right)$ exist, several discriminatory rules can be set. For example, we propose the following ones. Instead of defining $\beta_{k}\left(a_{D M}\right)$, one defines $B_{k}\left(a_{D M}\right) \in \Lambda_{k}\left(a_{D M}\right)$, the set of alternatives being the most similar to $a_{D M}$ on criterion $g_{k}$. The latter set is defined for any alternative $x$ as follows:

$$
B_{k}(x)=\underset{\forall a_{i} \in \Lambda_{k}(x)}{\arg \min } g_{k}\left(a_{i}\right)
$$

Several candidates in $B_{k}\left(a_{D M}\right)$ could exist. Therefore, one selects $B_{k}^{\prime}\left(a_{D M}\right)$, the set of candidates in $B_{k}\left(a_{D M}\right)$ that would be the most preferred if the worst scenario occurred, while trying to improve the performance of $a_{D M}$ on $g_{k}$. This set is defined for any alternative $x$ as follows:

$$
B_{k}^{\prime}(x)=\underset{\forall a_{i} \in B_{k}(x)}{\arg \max } \Delta_{k}\left(x, a_{i}\right)
$$

Again, there could be several candidates in $B_{k}^{\prime}\left(a_{D M}\right)$. Thus, one can find $\beta_{k}\left(a_{D M}\right)$, a set of observed alternatives that could be used as an intermediate benchmark and that cannot be distinguished in terms of similarity or in terms of a risk of degradation in performances. It is defined for any alternative $x$ as follows:

$$
\beta_{k}(x)=\underset{\forall a_{i} \in B_{k}^{\prime}(x)}{\arg \min } \Delta_{k}\left(a_{i}, x\right)
$$

This means that each candidate $a_{i} \in \beta_{k}\left(a_{D M}\right)$ minimizes the improvement on criteria different from $g_{k}$ on which it performs better than $a_{D M}$ (as any candidate in $B_{k}^{\prime}\left(a_{D M}\right)$ performs equally on $g_{k}$ ). This allows to increase the similarity between $\beta_{k}\left(a_{D M}\right)$ and $a_{D M}$ because Equation 16 minimizes the preference of $\beta_{k}\left(a_{D M}\right)$ over $a_{D M}$ on the set of criteria $J^{+}=\left\{g_{\ell} \in G \mid g_{\ell}\left(\beta_{k}\left(a_{D M}\right)\right)>g_{\ell}\left(a_{D M}\right) \cap g_{\ell} \neq g_{k}\right\}$.

\section{References}

Alirezaee, M., \& Afsharian, M. (2007). Model improvement for computational difficulties of DEA technique in the presence of special DMUs. Applied Mathematics and Computation, 186, 1600 - 1611.

Arrowsmith, J., Sisson, K., \& Marginson, P. (2004). What can 'benchmarking' offer the open method of co-ordination? Journal of European Public Policy, 11, 311-328. 
Banker, R. D., Charnes, A., \& Cooper, W. W. (1984). Some models for estimating technical and scale inefficiencies in data envelopment analysis. Management Science, 30, 1078-1092.

Behzadian, M., Kazemzadeh, R., Albadvi, A., \& Aghdasi, M. (2010). PROMETHEE: A comprehensive literature review on methodologies and applications. European Journal of Operational Research, 200, 198 -215 .

Billaut, J.-C., Bouyssou, D., \& Vincke, P. (2010). Should you believe in the Shanghai ranking? Scientometrics, 84, 237-263.

Bojković, N., Anić, I., \& Pejčić-Tarle, S. (2010). One solution for cross-country transport-sustainability evaluation using a modified ELECTRE method. Ecological Economics, 69, 1176 - 1186.

Borrás, S., \& Jacobsson, K. (2004). The open method of co-ordination and new governance patterns in the eu. Journal of European Public Policy, 11, 185-208.

Bovey, W. H., \& Hede, A. (2001). Resistance to organisational change: the role of defence mechanisms. Journal of Managerial Psychology, 16, 534-548.

Brans, J.-P., \& De Smet, Y. (2016). Promethee methods. In Multiple criteria decision analysis (pp. 187-219). Springer.

Camp, R. C. (1989). Benchmarking: The search for best practices that lead to superior performance. Quality Progress, 22, 70-75.

Charnes, A., Cooper, W., \& Rhodes, E. (1978). Measuring the efficiency of decision making units. European Journal of Operational Research, 2, $429-444$.

Ciomek, K., Ferretti, V., \& Kadziński, M. (2018). Predictive analytics and disused railways requalification: Insights from a post factum analysis perspective. Decision Support Systems, 105, 34-51.

Costa, C. A. B. E., \& Vansnick, J.-C. (1999). The MACBETH approach: Basic ideas, software, and an application. In Advances in decision analysis (pp. 131-157). Springer.

Dent, E. B., \& Goldberg, S. G. (1999). Challenging "resistance to change". The Journal of Applied Behavioral Science, 35, 25-41. 
Dutta, B., Singha, T., Goh, M., Lamata, M.-T., \& Verdegay, J.-L. (2019). Post factum analysis in TOPSIS based decision making method. Expert Systems with Applications, 138, 112806.

Estrada, S. A., Song, H. S., Kim, Y. A., Namn, S. H., \& Kang, S. C. (2009). A method of stepwise benchmarking for inefficient DMUs based on the proximity-based target selection. Expert Systems with Applications, 36, 11595-11604.

Figueira, J., Mousseau, V., \& Roy, B. (2005). ELECTRE methods. In Multiple Criteria Decision Analysis: State of the Art Surveys (pp. 133-153). Springer New York.

Georges, A., Vakaramoko, D., \& Hong, X. (2015). Application of multicriteria decision analysis in health care: a systematic review and bibliometric analysis. Health Expectations, 18, 1894-1905.

Ghahraman, A., \& Prior, D. (2016). A learning ladder toward efficiency: Proposing network-based stepwise benchmark selection. Omega, 63, $83-93$.

Hong, H. K., Ha, S. H., Shin, C. K., \& Sang (1999). Evaluating the efficiency of system integration projects using data envelopment analysis (DEA) and machine learning. Expert Systems with Applications, 16, 283 $-296$.

Howard, R. A. (1988). Decision analysis: practice and promise. Management science, 34, 679-695.

IOSC (2013). Principles for financial benchmarks. https://www.iosco.org/library/pubdocs/pdf/ $\backslash$ IOSCOPD409.pdf.

Kadziński, M., Ciomek, K., Rychły, P., \& Słowiński, R. (2016). Post factum analysis for robust multiple criteria ranking and sorting. Journal of Global Optimization, 65, 531-562.

Ligmann-Zielinska, A., \& Jankowski, P. (2012). Impact of proximity-adjusted preferences on rank-order stability in geographical multicriteria decision analysis. Journal of Geographical Systems, 14, 167-187.

Lim, S., Bae, H., \& Lee, L. H. (2011). A study on the selection of benchmarking paths in DEA. Expert Systems with Applications, 38, $7665-7673$.

Lootsma, F., Mensch, T., \& Vos, F. (1990). Multi-criteria analysis and budget reallocation in long-term research planning. European Journal of Operational Research, 47, $293-305$. 
Lozano, S., \& Calzada-Infante, L. (2017). Dominance network analysis of economic efficiency. Expert Systems with Applications, 82, $53-66$.

Lozano, S., \& Villa, G. (2005). Centralized DEA models with the possibility of downsizing. Journal of the Operational Research Society, 56, 357-364.

McNair, C., \& Leibfried, K. (1993). Benchmarking: A Tool for Continuous Improvement. Wiley.

Montibeller, G., \& Franco, L. A. (2011). Raising the bar: strategic multi-criteria decision analysis. Journal of the Operational Research Society, 62, 855-867.

Park, J. (2012). A DEA-based method of stepwise benchmark target selection with preference, direction and similarity criteria. International journal of innovative computing, information 8 control, 8.

Petrović, M., Bojković, N., \& Anić, I. (2012). Benchmarking the digital divide using a multi-level outranking framework: Evidence from EBRD countries of operation. Government Information Quarterly, 29, $597-607$.

Petrović, M., Bojković, N., Anić, I., Stamenković, M., \& Tarle, S. P. (2014). An ELECTRE-based decision aid tool for stepwise benchmarking: An application over EU digital agenda targets. Decision Support Systems, 59, $230-241$.

Petrović, M., Bojković, N., Stamenković, M., \& Anic, I. (2018a). A sensitivity analysis of ELECTRE based stepwise benchmarking for policy: The case of EU digital agenda. Management: Journal of Sustainable Business and Management Solutions in Emerging Economies, .

Petrović, M., Bojković, N., Stamenković, M., \& Anić, I. (2018b). Supporting performance appraisal in ELECTRE based stepwise benchmarking model. Omega, 78, 237 - 251.

Raman, R. (1997). Recent results on the single-source shortest paths problem. ACM SIGACT News, 28, $81-87$.

Roy, B. (1985). Méthodologie multicritère d'aide à la décision. Economica.

Roy, B. (1990). The outranking approach and the foundations of ELECTRE methods. In Readings in multiple criteria decision aid (pp. 155-183). Springer. 
Saaty, T. L., Vargas, L. G. et al. (2006). Decision making with the analytic network process volume 282. Springer.

Salo, A., Keisler, J., \& Morton, A. (2011). Portfolio decision analysis: improved methods for resource allocation volume 162. Springer Science \& Business Media.

Seiford, L. M., \& Zhu, J. (2003). Context-dependent data envelopment analysis - measuring attractiveness and progress. Omega, 31, $397-408$.

Steven, M. (2018). Towards benchmark stability and integrity. ICMA Annual Conference - Madrid, .

von Winterfeldt, D., \& Edwards, W. (1986). Decision Analysis and Behavioral Research. Cambridge, England: Cambridge University Press. 